\title{
Brainstem abnormalities and vestibular nerve enhancement in acute neuroborreliosis: a case report
}

Nadja A Farshad-Amacker, , Hans Scheffel, Thomas Frauenfelder and Hatem Alkadhi

\begin{abstract}
Background: Borreliosis is a widely distributed disease. Neuroborreliosis may present with unspecific symptoms and signs and often remains difficult to diagnose in patients with central nervous system symptoms, particularly if the pathognomonic erythema chronica migrans does not develop or is missed. Thus, vigilance is mandatory in cases with atypical presentation of the disease and with potentially severe consequences if not recognized early. We present a patient with neuroborreliosis demonstrating brain stem and vestibular nerve abnormalities on magnetic resonance imaging.

Case presentation: A 28-year-old Caucasian female presented with headaches, neck stiffness, weight loss, nausea, tremor, and gait disturbance. Magnetic resonance imaging showed T2-weighted hyperintense signal alterations in the pons and in the vestibular nerves as well as bilateral post-contrast enhancement of the vestibular nerves.

Serologic testing of the cerebrospinal fluid revealed the diagnosis of neuroborreliosis.

Conclusion: Patients infected with neuroborreliosis may present with unspecific neurologic symptoms and magnetic resonance imaging as a noninvasive imaging tool showing signal abnormalities in the brain stem and nerve root enhancement may help in establishing the diagnosis.
\end{abstract}

Keywords: Borreliosis, Brain stem, MRI, Neuroborreliosis, Vestibular nerve enhancement

\section{Background}

Borreliosis (Lyme disease) is a world wide distributed disease, transmitted by ticks, with particular focus in Europe and North America. Borreliosis can be classified in three clinical stages (Table 1). Only $40-60 \%$ of patients present with erythema chronicum migrans as an initial pathognomonic sign (stage I) [1]. Flu-like symptoms, such as fever, headaches, neck stiffness and arthralgia may be followed by meningeal irritation, encephalitis and/or neuritis (stage II) and further, cardiac involvement may develop. The final stage (stage III) may include arthritis, encephalomyelitis and acrodermatitis chronica atrophicans [2] (Table 1).

The diagnosis of neuroborreliosis is often challenging, particularly if the pathognomonic erythema chronicum migrans does not develop or is missed. Thus, it seems

\footnotetext{
* Correspondence: nadja.amacker@usz.ch

Diagnostic and Interventional Radiology Department, University Hospital of Zurich, Raemistrasse 100, Zurich 8091, Switzerland
}

emergent to call for vigilance in case of unspecific presentations of the disease which might have devastating consequences if not recognized and treated early. In neuroborreliosis, magnetic resonance imaging (MRI) may show meningeal and cranial nerve enhancement after contrast administration [3-5] and/or signal abnormalities in the white matter of the brain, although rarely reported [6].

We present a 28 -year-old patient infected with neuroborreliosis in whom MRI showed T2-weighted (T2w) hyperintense signal alterations in the pons and vestibular nerves as well as bilateral post-contrast vestibular nerve root enhancement.

\section{Case presentation}

A 28-year-old Caucasian female presented with headaches and fever of unknown origin lasting for two weeks. The patient noticed a tick bite two weeks prior to the onset of fever and headaches. Past history and family history were otherwise unremarkable. 
Table 1 Stages of borreliosis

\begin{tabular}{lll}
\hline Stages & Time course & Clinic manifestation \\
\hline Stage I & Days- weeks & Erythema chronicum migrans \\
Stage II & Weeks to $<6$ months & $\begin{array}{l}\text { Flue-like symptoms (Fever, arthralgias, } \\
\text { headaches, neck stiffness, } \\
\text { musculoskeletal pain, fatigue) }\end{array}$ \\
& & Early CNS- infection \\
& & (meningoencephalitis, neuritis) \\
& & $\begin{array}{l}\text { Cardiac involvement (AV- block, } \\
\text { peri- myocarditis) }\end{array}$ \\
& & Arthritis \\
Stage III $>6$ months & Encephalopathy (affecting mood, \\
& & memory and sleep) \\
& & Acrodermatitis chronica atrophicans \\
&
\end{tabular}

At the time of an ambulatory exam by a general practitioner (about three weeks after the tick bite), laboratory investigation e.g. complete blood cell count (CBC), Creactive protein $(\mathrm{CRP})$, electrolytes, liver enzymes, albumin, creatinin, lactate dehydrogenase (LDH), thyroid stimulating hormone (TSH), free thyroxine (free T4), glucose and Borrelia serum screening test were shown within normal limits. No abnormalities of the skin were noted. The patient was treated with non-steroidal analgetics and returned home.

The fever resolved spontaneously after two weeks (four weeks after the tick bite), however, the headaches remained and were further accompanied by neck stiffness, fatigue, nausea, weight loss of 5 pounds with decreased appetite, an intentional- and resting tremor, and diffuse disturbances in coarse coordination of the upper extremities. Finally, the patient developed sudden onset of immobilizing vertigo.

Eight weeks after the tick bite, blood laboratory evaluation was repeated. CBC, CRP, electrolytes, liver enzymes and creatinin were all still within normal limits. But at that time the results showed reactive borreliosis (Borrelia screening test: enzyme-linked immunosorbent assay (ELISA)-Immunoglobulin G (IgG)-antibody $>200 \mathrm{U} / \mathrm{ml}$ and ELISA-IgM-antibody $>100 \mathrm{U} / \mathrm{ml}$; normally each $<9 \mathrm{U} /$ $\mathrm{ml}$; Borrelia confirmation test: Western Blot-IgG and -IgM were also positive, reaction against $\mathrm{OspC}$ in IgM-antibodyclasses, and against $18 \mathrm{kD}, \mathrm{OspC}$ and VlsE in the IgGantibody-classes; Medica, Medical Laboratories Dr. F. Kaeppeli AG, Switzerland).

Lumbar puncture was subsequently performed. Cerebrospinal fluid (CSF) showed an increased protein count (2.006 g/l, normally $0.2-0.4 \mathrm{~g} / \mathrm{l})$, increased lactate level (2.5 mmol/l, normally $1.2-2.1 \mathrm{mmol} / \mathrm{l})$, slightly lowered glucose level ( $2 \mathrm{mmol} / \mathrm{l}$, normally $2.4-4.2 \mathrm{mmol} / \mathrm{l})$ and an increased lymphocyte (252 cells/ul, 99.5\% lymphocytes) and IgG-antibody count $(0.353 \mathrm{~g} / \mathrm{l}$, normally $<0.051 \mathrm{~g} / \mathrm{l})$. Further, CSF tests for viral agents such as early summer meningoencephalitis (ESME), human immunodeficiency virus (HIV), cytomegalovirus (CMV), enterovirus, epsteinbarr-virus (EBV), herpes simplex virus (HSV) and varicella zoster virus (VZV) were negative. However, reactive Borrelia burgdorferi IgG- and IgM-antibodies were positive using an enzyme immunoassay (EIA) test (Immunoblot IgG with recombinant antigens: $B$. burgdorferi VIsE and p41 positive and Immunoblot IgM with recombinant antigens: B. burgdorferi VIsE, p39, and OspC positive; Institute for Medical microbiology, University Hospital of Zurich, Switzerland).

An MRI of the brain was performed using a 1.5 Tesla MRI unit (Vision, Siemens, Medical Solutions, Erlangen, Germany). Mild hyperintense lesions on T2w TSE images were visible in the pons (Figure 1). Furthermore, strong bilateral T2w hyperintense signal alterations and postcontrast enhancement of the vestibular nerves within the

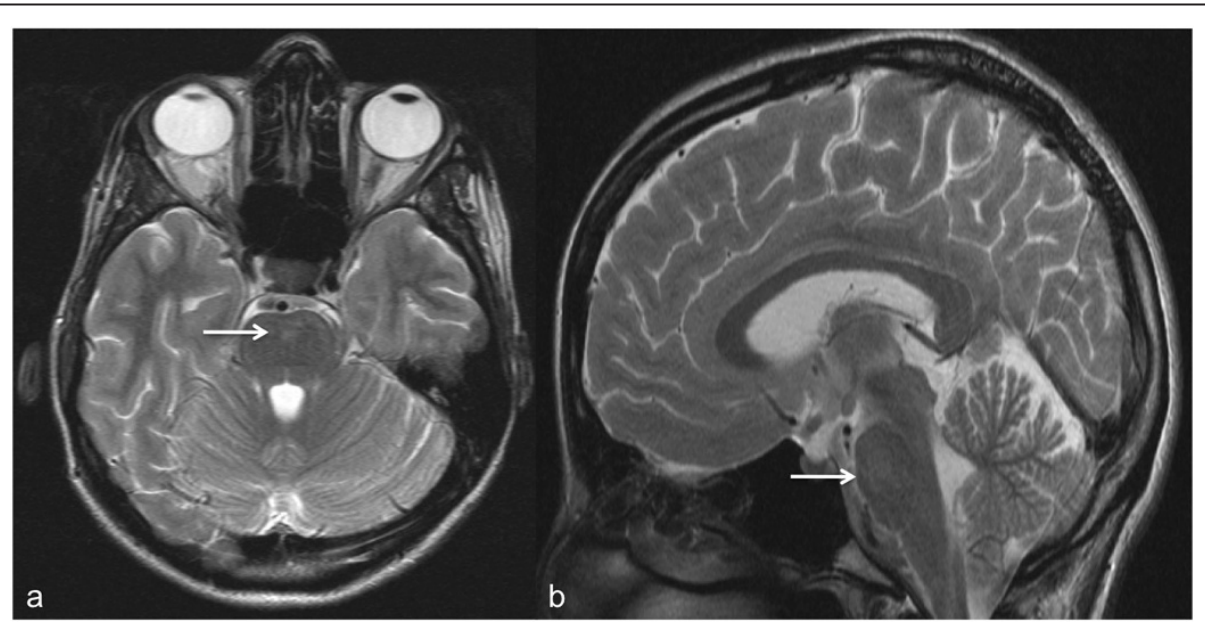

Figure 1 Brainstem abnormalities in a 28-year-old patient. (a) Axial and (b) sagittal T2w- TSE MR- images of the brain show hyperintense signal alterations in the pons (arrows). T2W, T2-weighted; TSE, turbo spin-echo; MR, magnetic resonance. 


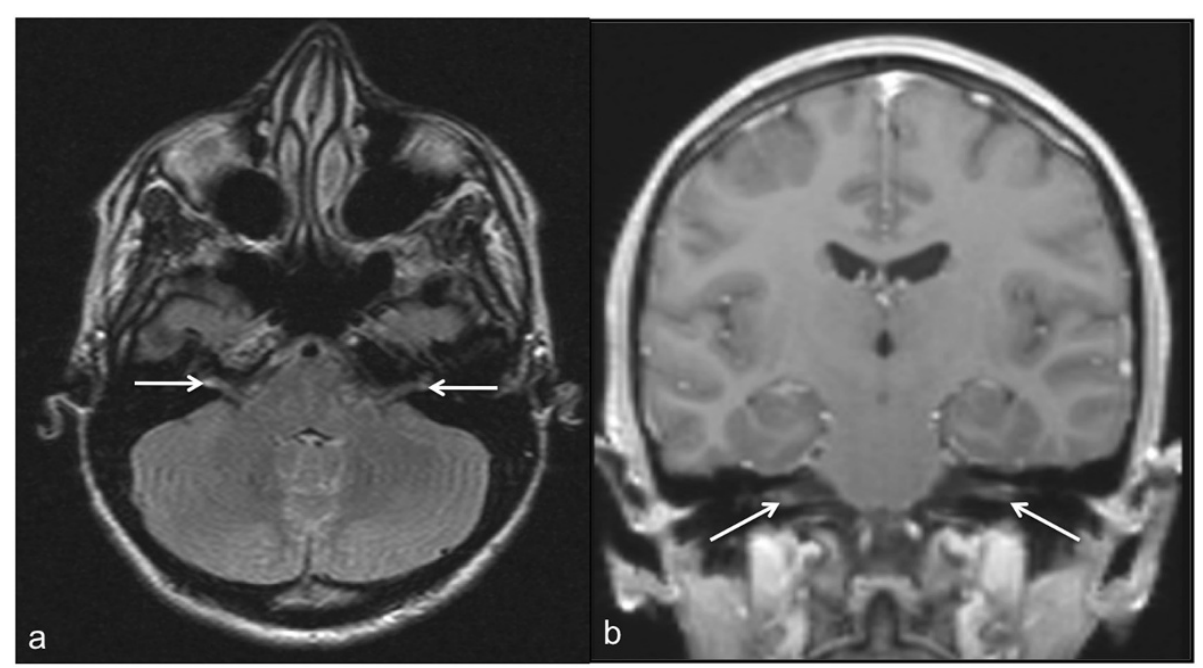

Figure 2 Hyperintense signal alterations and post-contrast vestibular nerve root enhancement in a 28 -year-old patient. (a) Axial T2W dark fluid image shows bilateral hyperintense signal in the vestibular nerves. (b) Coronal T1w, post-contrast image shows bilateral enhancement of the vestibular nerves.

internal auditory canal was noted (Figure 2). No meningeal enhancement, nor any diffusion restrictions were noted.

Therapy with intravenous ceftriaxone for three weeks was initiated. All symptoms resolved.

Here, we present a patient with hyperintense lesions in the pons and vestibular nerves as well as bilateral vestibular nerves post-contrast enhancement on MRI in a patient diseased with early stage II neuroborreliosis who presented with unspecific encephalopathic symptoms, without the characteristic erythema chronicum migrans and initially normal laboratory parameters but delayed positive reactive Borrelia IgM- and IgG-antibodies.

There are only a few cases reporting neuroborreliosis with brainstem abnormalities. In a recent case report, hyperintense lesions in the pons in a patient with neuroborreliosis having a 2-month history of neck pain, wasting, and fatigue followed by gait disturbance, dysarthria and dysmetria were shown [7]. Another report has described a case of borreliosis involving the brainstem in a 28-year-old man, showing symmetric patchy areas of hyperintensity involving the cerebellar peduncles eventually extending to the pons and cerebellar white matter [8]. Unfortunately, no contrast media was administered in this case [8].

Meningeal and/or cranial nerve enhancement has been reported thus so far. Right trigeminal nerve enhancement in a 15-year-old boy was previously described in a case report [5]. Further, simultaneous enhancement and thickening of the third and sixed cranial nerve [9] in a 57-year-old woman and also bilateral enhancement of cranial nerves III-V, as well as of cranial nerves VII and VIII on the left side in a 12-year-old girl [10] have been reported.
A retrospective study of 66 patients revealed that positive neuroimaging findings on MRI of patients with neuroborreliosis are relatively unusual and the authors concluded that findings are usually focal lesions in the white matter of the brain or nerve-root or meningeal enhancement [4]. Unlike previously reported studies, nerve-root or meningeal enhancement was detected in a substantial percentage of patients [4].

\section{Conclusion}

Patients with neuroborreliosis may present with unspecific neurologic symptoms and an MRI as a noninvasive imaging tool showing brain stem abnormalities and/or nerve root enhancement may help in establishing the diagnosis, especially since Borrelia antigens/antibodies are not routinely assessed in CSF. If the disease is missed and left untreated it might end in devastating consequences.

\section{Consent}

Written informed consent was obtained from the patient for the publication of this report and any accompanying images.

\section{Competing interest}

The authors declare that they have no competing interests.

\section{Authors' contributions}

All authors drafted and corrected the manuscript. All authors read and approved the final manuscript.

Received: 31 July 2012 Accepted: 16 December 2013

Published: 21 December 2013

\section{References}

1. Nadelman RB, Wormser GP: Erythema migrans and early Lyme disease. Am J Med 1995, 98:15S-23S. 
2. Nau R, Christen H-J, Eiffert H: Lyme disease-current state of knowledge. Disch Arztebl Int 2009, 106:72-81.

3. Nelson JA, Wolf MD, Yuh WT, Peeples ME: Cranial nerve involvement with Lyme borreliosis demonstrated by magnetic resonance imaging. Neurology 1992, 42:671-673.

4. Agarwal R, Sze G: Neuro-lyme disease: MR imaging findings. Radiology 2009, 253:167-173.

5. Köchling J, Freitag HJ, Bollinger T, Herz A, Sperner J: Lyme disease with lymphocytic meningitis, trigeminal palsy and silent thalamic lesion. Eur J Paediatr Neurol 2008, 12:501-504

6. Krüger H, Heim E, Schuknecht B, Scholz S: Acute and chronic neuroborreliosis with and without CNS involvement: a clinical, MRI, and HLA study of 27 cases. J Neurol 1991, 238:271-280.

7. Haene A, Troger M: Diffuse hyperintense brainstem lesions in neuroborreliosis. Neurology 2009, 73:326-326.

8. Kalina P, Decker A, Kornel E, Halperin JJ: Lyme disease of the brainstem. Neuroradiology 2005, 47:903-907.

9. Lell M, Schmid A, Stemper B, Maihöfner C, Heckmann JG, Tomandl BF: Simultaneous involvement of third and sixth cranial nerve in a patient with Lyme disease. Neuroradiology 2003, 45:85-87.

10. Huisman TA, Wohlrab G, Nadal D, Boltshauser E, Martin E: Unusual presentations of neuroborreliosis (Lyme disease) in childhood. J Comput Assist Tomogr 1999, 23:39-42.

doi:10.1186/1756-0500-6-551

Cite this article as: Farshad-Amacker et al:: Brainstem abnormalities and vestibular nerve enhancement in acute neuroborreliosis: a case report. BMC Research Notes 2013 6:551.

\section{Submit your next manuscript to BioMed Central and take full advantage of:}

- Convenient online submission

- Thorough peer review

- No space constraints or color figure charges

- Immediate publication on acceptance

- Inclusion in PubMed, CAS, Scopus and Google Scholar

- Research which is freely available for redistribution 